\title{
Multidimensional backward doubly stochastic differential equations with integral non-Lipschitz coefficients
}

\author{
Pengju Duan \\ School of Mathematics and Statistics, Suzhou University, Anhui, 234000, China. \\ Communicated by J. Brzdek
}

\begin{abstract}
The paper is devoted to solve multidimensional backward doubly stochastic differential equations under integral nonLipschitz conditions in general spaces. By stochastic analysis and constructing approximation sequence, a new set of sufficient conditions for multidimensional backward doubly stochastic differential equations is obtained. The results generalize the recent results on this issue. Finally, an example is given to illustrate the advantage of the main results. (c)2017 all rights reserved.
\end{abstract}

Keywords: Backward doubly stochastic differential equations, existence and uniqueness, integral non-Lipschitz. 2010 MSC: 60H10, 60H30, 60H99.

\section{Introduction}

Motivated by the probabilistic interpretation of solutions to a class of quasilinear parabolic partial differential equations (PDEs in short), Pardoux and Peng [14] introduced nonlinear backward stochastic differential equations (BSDEs in short). In the past decades, the theory of BSDEs have been extensively developed and gradually become an important tool in financial problems [2, 3], stochastic control [1] and stochastic games [7] and so on. One highlight of the theory is relaxing the conditions of existence and uniqueness of the solutions. Mao [13] has proved the existence and uniqueness of the multidimensional BSDEs with non-Lipschitz coefficients. Lepeltier and San Martin [10] have relaxed the generator with continuous conditions. Hamadène [6] investigated the existence of the multidimensional BSDEs where the generator satisfies uniformly continuous conditions. Recently, Fan et al. [5] discussed the existence and uniqueness of the multidimensional BSDEs with Osgood hypothesis where the method is different from [13]. Hu and Tang [9] studied the same problem with diagonally quadratic generators.

In 1994, Pardoux and Peng [15] studied the backward doubly stochastic differential equations (BDSDEs in short). They proved the existence and uniqueness under Lipschitz conditions, and also, discussed the probabilistic representation of solution of quasilinear stochastic PDEs. Furthermore, Shi et al. [16] obtained the existence of the BDSDEs with continuous coefficients. Lin [11, 12] made further efforts to establish the existence or uniqueness of solutions with non-Lipschitz. Even recently, Wang et al. [17]

Email address: pjduan1981@hotmail.com (Pengju Duan)

doi:10.22436/jnsa.010.01.16 
obtained the result where the first generator satisfied Osgood hypothesis, the second non-Lipschitz conditions. As a matter of fact, those results are obtained which the generators are uniform on $t$. To the best of our knowledge, the multidimensional BDSDEs with generators of integral non-Lipschitz assumptions in general spaces have rarely been reported.

The structure of this paper is organized as follows. In Section 2, we present some basic notions and assumptions which will be needed in the sequel. Section 3 is devoted to investigate the existence and uniqueness of solutions for multidimensional BDSDEs in general space. Finally, we give an example to show the effectiveness of the main result.

\section{Notations}

Let $T>0$ be a fixed terminal time. $|\cdot|$ denotes the Euclidean norm of $\mathbb{R}^{k}$, and $\langle x, y\rangle$ denotes the inner product of $x, y \in \mathbb{R}^{k}$. For any $z \in \mathbb{R}^{k \times d}$, its norm is defined by $\|z\|=\sqrt{\operatorname{Trace}\left(z z^{*}\right)}$. Let $(\Omega, \mathcal{F}, \mathrm{P})$ be a complete probability space, $\left\{B_{t}\right\}_{t \in[0, T]}$ and $\left\{W_{t}\right\}_{t \in[0, T]}$ are two mutually independent standard Brownian motions with values in $\mathbb{R}^{\mathrm{l}}$ and $\mathbb{R}^{\mathrm{d}}$, respectively, and $\mathcal{N}$ denotes the totality of P-null sets of $\mathcal{F}$. For any $\mathrm{t} \in[0, \mathrm{~T}]$,

$$
\mathcal{F}_{\mathrm{t}}=\mathcal{F}_{0, \mathrm{t}}^{W} \bigvee \mathcal{F}_{\mathrm{t}}^{\mathrm{B}} \text {, }
$$

where for any process $\eta_{t}, \mathcal{F}_{s, t}^{\eta}=\sigma\left\{\eta_{r}-\eta_{s}, s \leqslant r \leqslant t\right\} \vee \mathcal{N}, \mathcal{F}_{t}^{\eta}=\mathcal{F}_{0, t}^{\eta}$. For a deterministic square integrable function $a(t)$, we define $A(t)=\int_{0}^{t} a^{2}(s) d s$.

Let us introduce some spaces for $\beta>0$ which will be carried out in the following parts.

- $\mathrm{L}^{2}(\beta, a, T, \mathbb{R})$ denotes the set of all $\mathcal{F}_{\mathrm{T}}$-measurable $\mathbb{R}^{\mathrm{k}}$-valued random variables $\xi$ such that

$$
\|\xi\|^{2}=\mathbb{E}\left(e^{\beta A(T)}|\xi|^{2}\right)<+\infty .
$$

- $\mathrm{L}^{2}(\beta, a)$ denotes the collection of the $\mathcal{F}_{t}$-adapted, $\mathbb{R}^{k}$-valued continuous processes $\left(Y_{t}\right)_{t \in[0, T]}$ such that

$$
\|Y\|_{\beta}^{2}=\mathbb{E} \int_{0}^{T} e^{\beta A(t)}\left|Y_{t}\right|^{2} d t<+\infty
$$

- $\mathrm{L}^{2, a}(\beta, a)$ denotes the set of the $\mathcal{F}_{t}$-adapted, $\mathbb{R}^{k}$-valued continuous processes $\left(Y_{t}\right)_{t \in[0, T]}$ such that

$$
\|Y\|_{\beta, a}^{2}=\mathbb{E} \int_{0}^{T} e^{\beta A(t)} a^{2}(t)\left|Y_{t}\right|^{2} d t<+\infty
$$

- $\mathcal{S}^{2}(\beta, a)$ denotes the space of the $\mathcal{F}_{t}$-adapted, $\mathbb{R}^{k}$-valued continuous processes $\left(Y_{t}\right)_{t \in[0, T]}$ such that

$$
\|Y\|_{\mathcal{S}^{2}}^{2}=\mathbb{E}\left(\sup _{t \in[0, T]} e^{\beta A(t)}\left|Y_{t}\right|^{2}\right)<+\infty .
$$

- $\mathcal{M}^{2}(\beta, a)$ denotes the space of the $\mathcal{F}_{t}$-adapted, $\mathbb{R}^{\mathrm{k} \times \mathrm{d}}$-valued processes $\left(Z_{t}\right)_{t \in[0, T]}$ such that

$$
\|Z\|_{\mathcal{M}^{2}}^{2}=\mathbb{E} \int_{0}^{T} e^{\beta A(t)}\left\|Z_{t}\right\|^{2} d t<+\infty
$$

- $\mathcal{M}^{2, a}:=L^{2, a}(\beta, a) \times \mathcal{M}^{2}(\beta, a)$ denotes the Banach space with the norm

$$
\|Y, Z\|_{\beta}^{2}=\|Y\|_{\beta, a}^{2}+\|Z\|_{\mathcal{M}^{2}}^{2}
$$


- $\mathcal{M}^{2, c}:=\left(\mathcal{S}^{2}(\beta, a) \bigcap L^{2, a}(\beta, a)\right) \times \mathcal{M}^{2}(\beta, a)$ denotes the Banach space with the norm

$$
\|Y, Z\|_{\beta, c}^{2}=\|Y\|_{\mathcal{S}^{2}}^{2}+\|Y\|_{\beta, a}^{2}+\|Z\|_{\mathcal{M}^{2}}^{2} .
$$

In this paper, we consider the backward doubly stochastic differential equations

$$
Y_{t}=\xi+\int_{t}^{T} f\left(s, Y_{s}, Z_{s}\right) d s+\int_{t}^{T} g\left(s, Y_{s}, Z_{s}\right) d B_{s}-\int_{t}^{T} Z_{s} d W_{s}, t \in[0, T],
$$

where the integral with respect to $B_{t}$ is the classical backward Itô integral and the integral with respect to $W_{t}$ is standard forward Itô integral. The equations are often abbreviated by BDSDEs $(\xi, f, g)$.

With the above preparations, we introduce the definition of solution of (2.1).

Definition 2.1. A pair of processes $\left(Y_{t}, Z_{t}\right)_{t \in[0, T]} \in \mathcal{M}^{2, c}$ is a solution to (2.1), if it satisfies (2.1).

In order to get the solution of (2.1), we propose the following assumptions:

(H1) The terminal value $\xi \in \mathrm{L}^{2}(\beta, \mathrm{a}, \mathrm{T}, \mathbb{R})$;

(H2) (i) The coefficients $f: \Omega \times[0, \mathrm{~T}] \times \mathbb{R}^{\mathrm{k}} \times \mathbb{R}^{\mathrm{k} \times \mathrm{d}} \rightarrow \mathbb{R}^{\mathrm{k}}, \mathrm{g}: \Omega \times[0, \mathrm{~T}] \times \mathbb{R}^{\mathrm{k}} \times \mathbb{R}^{\mathrm{k} \times \mathrm{d}} \rightarrow \mathbb{R}^{\mathrm{k} \times \mathrm{l}}$, are progressively measurable for any $(y, z) \in \mathbb{R}^{k} \times \mathbb{R}^{k \times d}$ such that $\frac{f(\cdot, 0,0)}{a(\cdot)}, g(\cdot, 0,0) \in L^{2}(\beta, a)$.

(ii) There exist some integrable functions $p(t), q(t), u(t):[0, T] \rightarrow \mathbb{R}^{+}$such that for any $t \in$ $[0, T], y_{1}, y_{2} \in \mathbb{R}^{k}, z_{1}, z_{2} \in \mathbb{R}^{k \times d}$,

$$
\begin{aligned}
\left|f\left(t, y_{1}, z_{1}\right)-f\left(t, y_{2}, z_{2}\right)\right| & \leqslant p(t) \rho\left(\left|y_{1}-y_{2}\right|\right)+q(t)\left\|z_{1}-z_{2}\right\|, \\
\left\|g\left(t, y_{1}, z_{1}\right)-g\left(t, y_{2}, z_{2}\right)\right\|^{2} & \leqslant p(t)\left|y_{1}-y_{2}\right| \rho\left(\left|y_{1}-y_{2}\right|\right)+u(t)\left\|z_{1}-z_{2}\right\|^{2},
\end{aligned}
$$

where $\rho(x)$ is a concave and nondecreasing function with $\rho(0)=0$ and $\int_{0^{+}} \frac{d u}{\rho(u)}=+\infty$.

(iii) There exists a constant $0<\alpha<1$ such that $u(t) \leqslant \alpha$, for all $t \geqslant 0$.

Remark 2.2. For the above given spaces, if $\mathrm{a}(\mathrm{t})=\mathrm{C}, \mathrm{C}$ is a nonnegative constant, we can easily find that the spaces degenerate into the classical spaces.

Before giving our main results, we introduce some technical tools. The first lemma appears in [4].

Lemma 2.3. If $\rho(u)$ is a concave and nondecreasing function with $\rho(0)=0$ and $\int_{0^{+}} \frac{d u}{\rho(u)}=+\infty$, there exists a concave nondecreasing function $\phi(u)$ with $\phi(0)=0$ and $\int_{0^{+}} \frac{d u}{\phi(u)}=+\infty$, moreover, $a \sqrt{u} \rho(\sqrt{u}) \leqslant \phi(u) \leqslant$ $2 \mathrm{a} \sqrt{\mathrm{u}} \rho(\sqrt{\mathrm{u}})$, where $\mathrm{a}>0$ is a constant.

Lemma 2.4. Assume that the generator $\mathrm{f}$ satisfies $(\mathrm{H} 2)$. Let $\mathrm{f}^{(\mathfrak{i})}$ denote the $i$ th component of the generator $\mathrm{f}(\mathfrak{i}=$ $1,2, \cdots, k)$, we define a series of functions $\mathrm{f}_{\mathrm{n}}=\left(\mathrm{f}_{\mathrm{n}}^{(1)}, \mathrm{f}_{\mathrm{n}}^{(2)}, \cdots, \mathrm{f}_{\mathrm{n}}^{(\mathrm{k})}\right)$ with $\mathrm{f}_{\mathrm{n}}^{(\mathrm{i})}$ as follows

$$
f_{n}^{(i)}(t, y, z)=\inf _{u \in \mathbb{R}^{k}}\left\{f^{(i)}(t, u, z)+(n+A) p(t)|y-u|\right\} .
$$

Then, it satisfies

(i) for any $(y, z) \in \mathbb{R}^{k} \times \mathbb{R}^{k \times d},\left|f_{n}(t, y, z)-f(t, y, z)\right| \leqslant k p(t) \rho\left(\frac{2 A}{n}\right)$.

(ii) for any $\left(y_{i}, z_{i}\right) \in \mathbb{R}^{k} \times \mathbb{R}^{k \times d}, i=1,2$, we have

$$
\begin{gathered}
\left|f_{n}\left(t, y_{1}, z_{1}\right)-f_{n}\left(t, y_{2}, z_{2}\right)\right| \leqslant k(n+A)\left[p(t)\left|y_{1}-y_{2}\right|+q(t)\left\|z_{1}-z_{2}\right\|\right], \\
\left|f_{n}\left(t, y_{1}, z_{1}\right)-f_{n}\left(t, y_{2}, z_{2}\right)\right| \leqslant k p(t) \rho\left(\left|y_{1}-y_{2}\right|\right)+k q(t)\left\|z_{1}-z_{2}\right\| .
\end{gathered}
$$

(iii) $\frac{f_{n}(t, 0,0)}{a(t)} \in L^{2, a}(\beta, a)$.

Proof. The proof is similar to the process Step 1 of Theorem 1 in [5], so we omit it. 


\section{Existence and uniqueness}

In this section, we begin with establishing a priori estimate on the solutions of (2.1). Because $\rho(x)$ is a concave, there exists a nonnegative constant $A$ such that $\rho(x) \leqslant A(x+1)$. Furthermore, we let $a^{2}(t)=p(t)+q^{2}(t)$ in the following parts.

Proposition 3.1. Assume that (H1) and $(\mathrm{H} 2)$ hold, and $\left(\mathrm{Y}_{\mathrm{t}}, \mathrm{Z}_{\mathrm{t}}\right)$ is a solution of $(2.1)$, then for a large enough $\beta$, there exists a constant $\mathrm{d}_{\beta, \mathrm{T}}$ which depends on $\beta$ and $\mathrm{T}$ such that

$$
\begin{aligned}
& \mathbb{E}\left[\sup _{t \leqslant s \leqslant T} e^{\beta A(s)}\left|Y_{s}\right|^{2}\right]+\mathbb{E} \int_{t}^{T} e^{\beta A(s)} a^{2}(s)\left|Y_{s}\right|^{2} d s+\mathbb{E} \int_{t}^{T} e^{\beta A(s)}\left\|Z_{s}\right\|^{2} d s \\
& \leqslant d_{\beta, T}\left\{\mathbb{E} e^{\beta A(T)}|\xi|^{2}+\mathbb{E} \int_{t}^{T} e^{\beta A(s)} \frac{|f(s, 0,0)|^{2}}{a^{2}(s)} \mathrm{d} s+\mathbb{E} \int_{t}^{T} e^{\beta A(s)}|g(s, 0,0)|^{2} d s\right. \\
& \left.\quad+\mathbb{E} \int_{t}^{T} e^{\beta A(s)} a^{2}(s) \phi\left(\left|Y_{s}\right|^{2}\right) d s\right\} .
\end{aligned}
$$

Proof. Applying Itô formula to $e^{\beta A(t)}\left|Y_{t}\right|^{2}$, it yields that, for any $t \in[0, T]$

$$
\begin{aligned}
e^{\beta A(t)}\left|Y_{t}\right|^{2}+\beta \int_{t}^{T} e^{\beta A(s)} a^{2}(s)\left|Y_{s}\right|^{2} d s+\int_{t}^{T} e^{\beta A(s)}\left\|Z_{s}\right\|^{2} d s \\
=e^{\beta A(T)}|\xi|^{2}+2 \int_{t}^{T} e^{\beta A(s)} Y_{s} f\left(s, Y_{s}, Z_{s}\right) d s+2 \int_{t}^{T} e^{\beta A(s)} Y_{s} g\left(s, Y_{s}, Z_{s}\right) d B_{s} \\
\quad+\int_{t}^{T} e^{\beta A(s)}\left\|g\left(s, Y_{s}, Z_{s}\right)\right\|^{2} d s-2 \int_{t}^{T} e^{\beta A(s)} Y_{s} Z_{s} d W_{s} .
\end{aligned}
$$

Following the assumptions (H1) and (H2), and elementary inequality, we have

$$
\begin{aligned}
2 Y_{t} f\left(t, Y_{t}, Z_{t}\right) & =2 Y_{t}\left[f\left(t, Y_{t}, Z_{t}\right)-f\left(t, Y_{t}, 0\right)+f\left(t, Y_{t}, 0\right)-f(t, 0,0)+f(t, 0,0)\right] \\
& \leqslant 2\left|Y_{t}\right|\left|f\left(t, Y_{t}, Z_{t}\right)-f\left(t, Y_{t}, 0\right)\right|+2\left|Y_{t}\right|\left|f\left(t, Y_{t}, 0\right)-f(t, 0,0)\right|+2\left|Y_{t}\right||f(t, 0,0)| \\
& \leqslant\left(\frac{2}{1-\alpha}+\frac{\beta}{8}\right) a^{2}(t)\left|Y_{t}\right|^{2}+\frac{1-\alpha}{2}\left\|Z_{t}\right\|^{2}+a^{2}(t) \phi\left(\left|Y_{t}\right|^{2}\right)+\frac{8}{\beta a^{2}(t)}|f(t, 0,0)|^{2} \\
\left\|g\left(t, Y_{t}, Z_{t}\right)\right\|^{2} & =\left\|g\left(t, Y_{t}, Z_{t}\right)-g(t, 0,0)+g(t, 0,0)\right\|^{2} \\
& \leqslant \alpha\left(1+\frac{1}{\gamma}\right)\left\|Z_{t}\right\|^{2}+\left(1+\frac{1}{\gamma}\right) a^{2}(t) \phi\left(\left|Y_{t}\right|^{2}\right)+(1+\gamma)|g(t, 0,0)|^{2}
\end{aligned}
$$

where $\gamma$ is a nonnegative constant.

Take expectation on both sides of (3.1), by (3.2) and (3.3), we have

$$
\begin{aligned}
& \mathbb{E} e^{\beta A(t)}\left|Y_{t}\right|^{2}+\beta \mathbb{E} \int_{t}^{T} e^{\beta A(s)} a^{2}(s)\left|Y_{s}\right|^{2} d s+\mathbb{E} \int_{t}^{T} e^{\beta A(s)}\left\|Z_{s}\right\|^{2} d s \\
& \leqslant \mathbb{E} e^{\beta A(T)}|\xi|^{2}+\left(\frac{2}{1-\alpha}+\frac{\beta}{8}\right) \mathbb{E} \int_{t}^{T} e^{\beta A(s)} a^{2}(s)\left|Y_{s}\right|^{2} d s+\left[\frac{1-\alpha}{2}+\alpha\left(1+\frac{1}{\gamma}\right)\right] \mathbb{E} \int_{t}^{T} e^{\beta A(s)}\left\|Z_{s}\right\|^{2} d s \\
&+\frac{8}{\beta} \mathbb{E} \int_{t}^{T} e^{\beta A(s)} \frac{|f(s, 0,0)|^{2}}{a^{2}(s)} \mathrm{d} s+(1+\gamma) \mathbb{E} \int_{t}^{T} e^{\beta A(s)}|g(s, 0,0)|^{2} d s \\
&+\left(2+\frac{1}{\gamma}\right) \mathbb{E} \int_{t}^{T} e^{\beta A(s)} a^{2}(s) \phi\left(\left|Y_{s}\right|^{2}\right) d s .
\end{aligned}
$$


Let $\gamma=\frac{4 \alpha}{1-\alpha}$, we deduce

$$
\begin{aligned}
& \mathbb{E} e^{\beta A(t)}\left|Y_{t}\right|^{2}+\left(\frac{7 \beta}{8}-\frac{2}{1-\alpha}\right) \mathbb{E} \int_{t}^{T} e^{\beta A(s)} a^{2}(s)\left|Y_{s}\right|^{2} d s+\frac{1-\alpha}{4} \mathbb{E} \int_{t}^{T} e^{\beta A(s)}\left\|Z_{s}\right\|^{2} d s \\
& \leqslant \mathbb{E} e^{\beta A(T)}|\xi|^{2}+(1+\gamma) \mathbb{E} \int_{t}^{T} e^{\beta A(s)}|g(s, 0,0)|^{2} d s+\frac{8}{\beta} \mathbb{E} \int_{t}^{T} e^{\beta A(s)} \frac{|f(s, 0,0)|^{2}}{a^{2}(s)} d s \\
& \quad+\left(2+\frac{1}{\gamma}\right) \mathbb{E} \int_{t}^{T} e^{\beta A(s)} a^{2}(s) \phi\left(\left|Y_{s}\right|^{2}\right) d s .
\end{aligned}
$$

Let $\beta$ be large enough, there exists a nonnegative constant $C_{\beta, T}$ such that

$$
\mathbb{E} e^{\beta A(t)}\left|Y_{t}\right|^{2}+\mathbb{E} \int_{t}^{T} e^{\beta A(s)} a^{2}(s)\left|Y_{s}\right|^{2} d s+\mathbb{E} \int_{t}^{T} e^{\beta A(s)}\left\|Z_{s}\right\|^{2} d s \leqslant C_{\beta, T} X_{t} .
$$

where

$$
X_{t}=\mathbb{E} e^{\beta A(T)}|\xi|^{2}+\mathbb{E} \int_{t}^{T} e^{\beta A(s)} \frac{|f(s, 0,0)|^{2}}{a^{2}(s)} d s+\mathbb{E} \int_{t}^{T} e^{\beta A(s)}|g(s, 0,0)|^{2} d s+\mathbb{E} \int_{t}^{T} e^{\beta A(s)} a^{2}(s) \phi\left(\left|Y_{s}\right|^{2}\right) d s .
$$

By the Burkholder-Davis-Gundy inequality, we have

$$
\begin{aligned}
& 2 \mathbb{E}\left[\sup _{r \in[t, T]}\left|\int_{r}^{T} e^{\beta A(s)} Y_{s} g\left(s, Y_{s}, Z_{s}\right) d B_{s}\right|\right] \leqslant 12 \mathbb{E}\left[\sup _{r \in[t, T]}\left[e^{\frac{\beta A(r)}{2}} Y_{r}\right]\left(\int_{t}^{T} e^{\beta A(s)}\left\|g\left(s, Y_{s}, Z_{s}\right)\right\|^{2} d s\right)^{1 / 2}\right] \\
& \leqslant \frac{1}{4} \mathbb{E}\left[\sup _{r \in[t, T]}\left[e^{\beta A(r)}\left|Y_{r}\right|^{2}\right]\right]+144 \mathbb{E} \int_{t}^{T} e^{\beta A(s)} \| g\left(s, Y_{s}, Z_{s} \|^{2} d s .\right. \\
& 2 \mathbb{E}\left[\sup _{r \in[t, T]}\left|\int_{s}^{T} e^{\beta A(s)} Y_{s} Z_{s} d W_{s}\right|\right] \leqslant \frac{1}{4} \mathbb{E}\left\{\sup _{r \in[t, T]}\left[e^{\beta A(r)}\left|Y_{r}\right|^{2}\right]\right\}+144 \mathbb{E} \int_{r}^{T} e^{\beta A(s)}\left\|Z_{s}\right\|^{2} d s .
\end{aligned}
$$

From (3.1), (3.5), and (3.6), it follows that

$$
\begin{aligned}
& \mathbb{E}\left[\sup _{r \in[\mathrm{t}, T]} e^{\beta A(r)}\left|Y_{r}\right|^{2}\right]+\beta \mathbb{E} \int_{t}^{T} e^{\beta A(s)} a^{2}(s)\left|Y_{s}\right|^{2} d s+\mathbb{E} \int_{t}^{T} e^{\beta A(s)}\left\|Z_{s}\right\|^{2} d s \\
& \leqslant \mathbb{E} e^{\beta A(T)}|\xi|^{2}+2 \mathbb{E} \int_{t}^{T} e^{\beta A(s)} Y_{s} f\left(s, Y_{s}, Z_{s}\right) d s+\int_{t}^{T} e^{\beta A(s)}\left\|g\left(s, Y_{s}, Z_{s}\right)\right\|^{2} d s \\
&+2 \mathbb{E}\left[\left|\sup _{r \in[t, T]} \int_{r}^{T} e^{\beta A(s)} Y_{s} g\left(s, Y_{s}, Z_{s}\right) d B_{s}\right|\right]+2 \mathbb{E}\left\{\sup _{r \in[t, T]}\left|\int_{r}^{T} e^{\beta A(s)} Y_{s} Z_{s} d W_{s}\right|\right\} \\
& \leqslant \mathbb{E} e^{\beta A(T)}|\xi|^{2}+\frac{1}{2} \mathbb{E}\left[\sup _{r \in[t, T]} e^{\beta A(r)}\left|Y_{r}\right|^{2}\right]+145(1+\gamma) \mathbb{E} \int_{t}^{T} e^{\beta A(s)}|g(s, 0,0)|^{2} d s \\
&+\left[144+\frac{1-\alpha}{2}+145 \alpha\left(1+\frac{1}{\gamma}\right)\right] \mathbb{E} \int_{t}^{T} e^{\beta A(s)}\left\|Z_{s}\right\|^{2} d s \\
&+\frac{8}{\beta} \mathbb{E} \int_{t}^{T} e^{\beta A(s)} \frac{|f(s, 0,0)|^{2}}{a^{2}(s)} d s+\left(\frac{2}{1-\alpha}+\frac{\beta}{8}\right) \mathbb{E} \int_{t}^{T} e^{\beta A(s)} a^{2}(s)\left|Y_{s}\right|^{2} d s \\
&+\left(146+\frac{145}{\gamma}\right) \mathbb{E} \int_{t}^{T} e^{\beta A(s)} a^{2}(s) \phi\left(\left|Y_{s}\right|^{2}\right) d s .
\end{aligned}
$$

From (3.4) and (3.7), we can derive the result. 
Theorem 3.2. Assume (H1) and ( $\mathrm{H} 2)$ hold. Then, there exists a unique solution $\left(\mathrm{Y}_{\mathrm{t}}, \mathrm{Z}_{\mathrm{t}}\right) \in \mathcal{M}^{2, \mathrm{c}}$ satisfying (2.1).

Proof. Uniqueness. Let $\left(Y_{t}^{i}, Z_{t}^{i}\right) \in \mathcal{M}^{2, c}(i=1,2)$ be solutions of $(2.1)$, we have

$$
Y_{t}^{1}-Y_{t}^{2}=\int_{t}^{T}\left[f\left(s, Y_{s}^{1}, Z_{s}^{1}\right)-f\left(s, Y_{s}^{2}, Z_{s}^{2}\right)\right] d s+\int_{t}^{T}\left[g\left(s, Y_{s}^{1}, Z_{s}^{1}\right)-g\left(s, Y_{s}^{2}, Z_{s}^{2}\right)\right] d B_{s}-\int_{t}^{T}\left(Z_{s}^{1}-Z_{s}^{2}\right) d W_{s} .
$$

Applying Itô formula to $e^{\beta A(t)}\left|Y_{t}^{1}-Y_{t}^{2}\right|^{2}$,

$$
\begin{aligned}
e^{\beta A(t)} & \left|Y_{t}^{1}-Y_{t}^{2}\right|^{2}+\beta \int_{t}^{T} e^{\beta A(s)} a^{2}(s)\left|Y_{s}^{1}-Y_{s}^{2}\right|^{2} d s+\int_{t}^{T} e^{\beta A(s)}\left\|Z_{s}^{1}-Z_{s}^{2}\right\|^{2} d s \\
= & 2 \int_{t}^{T} e^{\beta A(s)}\left(Y_{s}^{1}-Y_{s}^{2}\right)\left[f\left(s, Y_{s}^{1}, Z_{s}^{1}\right)-f\left(s, Y_{s}^{2}, Z_{s}^{2}\right)\right] d s \\
& +\int_{t}^{T} e^{\beta A(s)} \|\left(g\left(s, Y_{s}^{1}, Z_{s}^{1}\right)-g\left(s, Y_{s}^{2}, Z_{s}^{2}\right) \|^{2} d s-2 \int_{t}^{T} e^{\beta A(s)}\left(Y_{s}^{1}-Y_{s}^{2}\right)\left(Z_{s}^{1}-Z_{s}^{2}\right) d W_{s}\right. \\
& +2 \int_{t}^{T} e^{\beta A(s)}\left(Y_{s}^{1}-Y_{s}^{2}\right)\left[g\left(s, Y_{s}^{1}, Z_{s}^{1}\right)-g\left(s, Y_{s}^{2}, Z_{s}^{2}\right)\right] d B_{s} .
\end{aligned}
$$

Taking expectation on both sides of (3.8), from (H1), (H2) and elementary inequality $2 a b \leqslant \theta a^{2}+\frac{1}{\theta} b^{2}, \theta>$ 0 , we have

$$
\begin{aligned}
& \mathbb{E} e^{\beta A(t)}\left|Y_{t}^{1}-Y_{t}^{2}\right|^{2}+\beta \mathbb{E} \int_{t}^{T} e^{\beta A(s)} a^{2}(s)\left|Y_{s}^{1}-Y_{s}^{2}\right|^{2} d s+\mathbb{E} \int_{t}^{T} e^{\beta A(s)}\left\|Z_{s}^{1}-Z_{s}^{2}\right\|^{2} d s \\
&=2 \mathbb{E} \int_{t}^{T} e^{\beta A(s)}\left(Y_{s}^{1}-Y_{s}^{2}\right)\left[f\left(s, Y_{s}^{1}, Z_{s}^{1}\right)-f\left(s, Y_{s}^{2}, Z_{s}^{2}\right)\right] d s \\
& \quad+\mathbb{E} \int_{t}^{T} e^{\beta A(s)}\left\|g\left(s, Y_{s}^{1}, Z_{s}^{1}\right)-g\left(s, Y_{s}^{2}, Z_{s}^{2}\right)\right\|^{2} d s \\
& \leqslant 3 \mathbb{E} \int_{t}^{T} e^{\beta A(s)} a^{2}(s) \phi\left(\left|Y_{s}^{1}-Y_{s}^{2}\right|^{2}\right) d s+\frac{\beta}{4} \mathbb{E} \int_{t}^{T} e^{\beta A(s)} a^{2}(s)\left|Y_{s}^{1}-Y_{s}^{2}\right|^{2} d s \\
&+\left(\frac{4}{\beta}+\alpha\right) \mathbb{E} \int_{t}^{T} e^{\beta A(s)}\left\|Z_{s}^{1}-Z_{s}^{2}\right\|^{2} d s .
\end{aligned}
$$

By Lemma 2.3, and taking $\beta$ large enough, there exists a nonnegative constant $C_{1}$ such that

$$
\mathbb{E} \int_{t}^{T} e^{\beta A(s)} a^{2}(s)\left|Y_{s}^{1}-Y_{s}^{2}\right|^{2} d s+\mathbb{E} \int_{t}^{T} e^{\beta A(s)}\left\|Z_{s}^{1}-Z_{s}^{2}\right\|^{2} d s \leqslant C_{1} \mathbb{E} \int_{t}^{T} e^{\beta A(s)} a^{2}(s) \phi\left(\left|Y_{s}^{1}-Y_{s}^{2}\right|^{2}\right) d s .
$$

From (3.8), (3.9) and Burkholder-Davis-Gundy inequality, there exists a nonnegative constant $\mathrm{C}_{2}$ such that

$$
\begin{gathered}
\mathbb{E}\left[\sup _{t \leqslant r \leqslant T} e^{\beta A(r)}\left|Y_{r}^{1}-Y_{r}^{2}\right|^{2}\right]+\mathbb{E} \int_{t}^{T} e^{\beta A(s)} a^{2}(s)\left|Y_{s}^{1}-Y_{s}^{2}\right|^{2} d s+\mathbb{E} \int_{t}^{T} e^{\beta A(s)}\left\|Z_{s}^{1}-Z_{s}^{2}\right\|^{2} d s \\
\leqslant C_{2} \mathbb{E} \int_{t}^{T} e^{\beta A(s)} a^{2}(s) \phi\left(\left|Y_{s}^{1}-Y_{s}^{2}\right|^{2}\right) d s \leqslant C_{2} \int_{t}^{T} a^{2}(s) \phi\left(\mathbb{E} \sup _{s \leqslant r \leqslant T} e^{\beta A(r)}\left|Y_{r}^{1}-Y_{r}^{2}\right|^{2}\right) d s .
\end{gathered}
$$

By Bihari inequality, we can obtain $Y_{s}^{1}=Y_{s}^{2}, Z_{s}^{1}=Z_{s}^{2}, d P-$ a.s.

Existence. By the definition of $f_{n}$, Lemma 2.3, and Lemma 2.4, we can easily deduce BDSDEs $\left(\xi, f_{n}, g\right)$ is a special case in [8]. Therefore, $\operatorname{BDSDEs}\left(\xi, f_{n}, g\right)$ have a unique solution denoted by $\left(Y_{t}^{n}, Z_{t}^{n}\right)$. Applying Itô formula to $e^{\beta A(t)}\left|Y_{t}^{n}-Y_{t}^{m}\right|^{2}$,

$$
e^{\beta A(t)}\left|Y_{t}^{n}-Y_{t}^{m}\right|^{2}+\beta \int_{t}^{T} e^{\beta A(s)} a^{2}(s)\left|Y_{s}^{n}-Y_{s}^{m}\right|^{2} d s+\int_{t}^{T} e^{\beta A(s)}\left\|Z_{s}^{n}-Z_{s}^{m}\right\|^{2} d s
$$




$$
\begin{aligned}
= & 2 \int_{t}^{T} e^{\beta A(s)}\left(Y_{s}^{n}-Y_{s}^{m}\right)\left[f_{n}\left(s, Y_{s}^{n}, Z_{s}^{n}\right)-f_{m}\left(s, Y_{s}^{m}, Z_{s}^{m}\right)\right] d s \\
& +2 \int_{t}^{T} e^{\beta A(s)}\left(Y_{s}^{n}-Y_{s}^{m}\right)\left[g\left(s, Y_{s}^{n}, Z_{s}^{n}\right)-g\left(s, Y_{s}^{m}, Z_{s}^{m}\right)\right] d B_{s} \\
& -2 \int_{t}^{T} e^{\beta A(s)}\left(Y_{s}^{n}-Y_{s}^{m}\right)\left(Z_{s}^{n}-Z_{s}^{m}\right) d W_{s} \\
& +\int_{t}^{T} e^{\beta A(s)} \|\left(g\left(s, Y_{s}^{n}, Z_{s}^{n}\right)-g\left(s, Y_{s}^{m}, Z_{s}^{m}\right) \|^{2} d s .\right.
\end{aligned}
$$

Taking suitable $\beta$, by Lemma 2.3 and Burkholder-Davis-Gundy inequality, there exists a nonnegative constant $C_{3}$ such that

$$
\begin{aligned}
& \mathbb{E}\left[\sup _{t \leqslant r \leqslant T} e^{\beta A(r)}\left|Y_{r}^{n}-Y_{r}^{m}\right|^{2}\right]+\mathbb{E} \int_{t}^{T} e^{\beta A(s)} a^{2}(s)\left|Y_{s}^{n}-Y_{s}^{m}\right|^{2} d s+\mathbb{E} \int_{t}^{T} e^{\beta A(s)}\left\|Z_{s}^{n}-Z_{s}^{m}\right\|^{2} d s \\
& \quad \leqslant C_{3} \mathbb{E} \int_{t}^{T} e^{\beta A(s)} a^{2}(s) \psi\left(\left|Y_{s}^{n}-Y_{s}^{m}\right|^{2}\right) d s \\
& \quad \leqslant C_{3} \int_{t}^{T} a^{2}(s) \psi\left(\mathbb{E} \sup _{s \leqslant r \leqslant T} e^{\beta A(r)}\left|Y_{r}^{n}-Y_{r}^{m}\right|^{2}\right) d s,
\end{aligned}
$$

where $\psi(u)$ is a concave and nondecreasing function with $\psi(0)=0$ and $\int_{0^{+}} \frac{d u}{\psi(u)}=+\infty, k u \rho(u) \leqslant \psi(u) \leqslant$ $2 k u \rho(u), k>0$.

From Bihari inequality and (3.10), we have $\left(Y_{t}^{n}, Z_{t}^{n}\right)$ is a Cauchy sequence in $\mathcal{M}^{2, c}$.

On the other hand,

$$
\begin{aligned}
& \int_{t}^{T} e^{\beta A(s)}\left|f_{n}\left(s, Y_{s}^{n}, Z_{s}^{n}\right)-f\left(s, Y_{s}, Z_{s}\right)\right| d s \\
& \leqslant \mathbb{E} \int_{t}^{T} e^{\beta A(s)}\left[k p(s) \rho\left(\frac{2 A}{n}\right)+p(s) \rho\left(\left|Y_{s}^{n}-Y_{s}\right|\right)+q(s)\left\|Z_{s}^{n}-Z_{s}\right\|\right] d s \\
& \leqslant \mathbb{E} \int_{t}^{T} e^{\beta A(s)} k p(s)\left[\rho\left(\frac{2 A}{n}\right)+\rho\left(\frac{2 A}{m+A}\right)\right] d s+(m+A) \mathbb{E} \int_{t}^{T} e^{\beta A(s)} p(s)\left|Y_{s}^{n}-Y_{s}\right| d s \\
& +\mathbb{E} \int_{t}^{T} e^{\beta A(s)} q(s)\left\|Z_{s}^{n}-Z_{s}\right\| d s \\
& \leqslant \mathbb{E} \int_{t}^{T} e^{\beta A(s)} k p(s)\left[\rho\left(\frac{2 A}{n}\right)+\rho\left(\frac{2 A}{m+A}\right)\right] d s \\
& +\mathbb{E}\left\{\left[\int_{t}^{T} e^{\beta A(s)} a^{2}(s) d s\right]^{\frac{1}{2}}\left[\int_{t}^{T} e^{\beta A(s)}\left\|Z_{s}^{n}-Z_{s}\right\|^{2} d s\right]^{\frac{1}{2}}\right\} \\
& +(m+A) \mathbb{E}\left\{\left[\int_{t}^{T} e^{\beta A(s)} a^{2}(s) d s\right]^{\frac{1}{2}}\left[\int_{t}^{T} e^{\beta A(s)} a^{2}(s)\left|Y_{s}^{n}-Y_{s}\right|^{2} d s\right]^{\frac{1}{2}}\right\}, \\
& \mathbb{E} \int_{t}^{T} e^{\beta A(s)}\left\|g_{n}\left(s, Y_{s}^{n}, Z_{s}^{n}\right)-g\left(s, Y_{s}, Z_{s}\right)\right\|^{2} d s \\
& \leqslant \mathbb{E} \int_{t}^{T} e^{\beta A(s)}\left[\left|Y_{s}^{n}-Y_{s}\right| p(s) \rho\left(\left|Y_{s}^{n}-Y_{s}\right|\right)\right] d s+\alpha \mathbb{E} \int_{t}^{T} e^{\beta A(s)}\left\|Z_{s}^{n}-Z_{s}\right\|^{2} d s \\
& \leqslant \int_{t}^{T} p(s) \rho\left(\mathbb{E}\left[\sup _{s \leqslant r \leqslant T} e^{\beta A(r)}\left|Y_{r}^{n}-Y_{r}\right|^{2}\right)\right] d s+\alpha \mathbb{E} \int_{t}^{T} e^{\beta A(s)}\left\|Z_{s}^{n}-Z_{s}\right\|^{2} d s .
\end{aligned}
$$


From (3.11) and (3.12), we have

$$
Y_{t}=\xi+\int_{t}^{T} f\left(s, Y_{s}, Z_{s}\right) d s+\int_{t}^{T} g\left(s, Y_{s}, Z_{s}\right) d B_{s}-\int_{t}^{T} Z_{s} d W_{s}, t \in[0, T]
$$

Then, $\left(Y_{t}, Z_{t}\right)_{t \in[0, T]}$ is a solution of (2.1).

Example 3.3. For convenience, let $k=1$, and $f(t, y, z)=\frac{1}{\sqrt{t}} h(|y|)+\frac{1}{\sqrt[6]{t}}|z|+\left|B_{t}\right|, g(t, y, z)=\frac{1}{\sqrt[4]{t}} \sin |y|+$ $\frac{1}{2 \sqrt{2+t^{2}}}|z|+\left|B_{t}\right|$, and $\delta$ is a enough small nonnegative constant,

$$
h(x)= \begin{cases}-x \ln x, & x \leqslant \delta \\ h^{\prime}(\delta-)(x-\delta)+h(\delta), & x>\delta \\ 0, & \text { other cases. }\end{cases}
$$

We choose $p(t)=\frac{1}{\sqrt{t}}, q(t)=\frac{1}{\sqrt[6]{t}}, u(t)=\frac{1}{2+t^{2}}$, then

$$
\begin{gathered}
\left|f\left(t, y_{1}, z_{1}\right)-f\left(t, y_{2}, z_{2}\right)\right| \leqslant p(t) h\left(\left|y_{1}-y_{2}\right|\right)+q(t)\left\|z_{1}-z_{2}\right\|, \\
\left|g\left(t, y_{1}, z_{1}\right)-g\left(t, y_{2}, z_{2}\right)\right|^{2} \leqslant\left[\frac{1}{\sqrt[4]{t}}\left|y_{1}-y_{2}\right|+\frac{1}{2 \sqrt{2+t^{2}}}|| z_{1}-z_{2} \|\right]^{2} \leqslant 3 p(t)\left|y_{1}-y_{2}\right|^{2}+\frac{1}{2+t^{2}}\left\|z_{1}-z_{2}\right\|^{2} .
\end{gathered}
$$

Let $\rho(x)=h(x)+3 x$, we can deduce $\rho(x)$ is a concave function, $\int_{0^{+}} \frac{1}{\rho(x)} d x=+\infty$. According to above analysis, the functions $f(t, y, z), g(t, y, z)$ satisfy $(H 1)$ and $(H 2)$, the equation (2.1) has a unique solution. Obviously, $f(t, y, z), g(t, y, z)$ do not satisfy the assumptions in $[11,12,15-17]$. Let $p(t), q(t)=C, u(t)=$ $\alpha(0<\alpha<1), C$ is a nonnegative constant, then, the results generalize the results in [15, 17]. Moreover, if $g(t, y, z)=0,[5,13,14]$ are special cases of our main results.

\section{Acknowledgment}

The author would like to thank his/her referee for the valuable comments. The work is partially supported by key program of study home and abroad for young scholar sponsored by Anhui Province (gxfxZD2016261), the project for outstanding academic and technical backbone of Suzhou University (2014xjgg05), and Natural Science Foundation of Anhui Province (1508085MA10).

\section{References}

[1] N. El-Karoui, S. Hamadéne, BSDEs and risk-sensitive control, zero-sum and nonzero-sum game problems of stochastic functional differential equations, Stochastic Process. Appl., 107 (2003), 145-169. 1

[2] N. El-Karoui, S. Peng, M. C. Quenez, Backward stochastic differential equations in finance, Math. Finance, 7 (1997), 1-71. 1

[3] N. El-Karoui, M. C. Quenez, Non-linear pricing theory and backward stochastic differential equations, Financial mathematics, Bressanone, (1996), 191-246, Lecture Notes in Math., 1656, Springer, Berlin, (1997). 1

[4] S.-J. Fan, L. Jiang, Multidimensional BSDEs with weak monotonicity and general growth generators, Acta Math. Sin. (Engl. Ser.), 23 (2013), 1885-1906. 2

[5] S.-J. Fan, L. Jiang, M. Davison, Existence and uniqueness result for multidimensional BSDEs with generators of Osgood type, Front. Math. China, 4 (2013), 811-824. 1, 2, 3.3

[6] S. Hamadène, Multidimensional backward stochastic differential equations with uniformly continuous coefficients, Bernoulli, 9 (2003), 517-534. 1

[7] S. Hamadène, J.-P. Lepeltier, Zero-sum stochastic differential games and backward equations, Systems Control Lett., 24 (1995), 259-263. 1

[8] L.-Y. Hu, Reflected backward doubly stochastic differential equations driven by a Lévy process with stochastic Lipschitz condition, Appl. Math. Comput., 219 (2012), 1153-1157. 3

[9] Y. Hu, S.-J. Tang, Multi-dimensional backward stochastic differential equations of diagonally quadratic generators, Stochastic Process. Appl., 126 (2016), 1066-1086. 1

[10] J. P. Lepeltier, J. San Martin, Backward stochastic differential equations with continuous coefficient, Statist. Probab. Lett, 32 (1997), 425-430. 1 
[11] Q. Lin, A class of backward doubly stochastic differential equations with non-Lipschitz coefficients, Statist. Probab. Lett., 79 (2009), 2223-2229. 1, 3.3

[12] Q. Lin, A generalized existence theorem of backward doubly stochastic differential equations, Acta Math. Sin. (Engl. Ser.), 26 (2010), 1525-1534. 1, 3.3

[13] X.-R. Mao, Adapted solutions of backward stochastic differential equations with non-Lipschitz coefficients, Stochastic Process. Appl., 58 (1995), 281-292. 1, 3.3

[14] É. Pardoux, S. G. Peng, Adapted solution of a backward stochastic differential equation, Systems Control Lett., 14 (1990), 55-61. 1, 3.3

[15] É. Pardoux, S. G. Peng, Backward doubly stochastic differential equations and systems of quasilinear SPDEs, Probab. Theory Related Fields, 98 (1994), 209-227. 1, 3.3

[16] Y.-F. Shi, Y.-L. Gu, K. Liu, Comparison theorems of backward doubly stochastic differential equations and applications, Stoch. Anal. Appl., 23 (2005), 97-110. 1

[17] X. F. Wang, L. Jiang, J. J. Ma, Multidimensional backward doubly stochastic differential equations with generators of Osgood type, (Chinese) J. Shandong Univ. Nat. Sci., 50 (2015), 24-33. 1, 3.3 